\title{
Scattering Matrix Formulation of the Topological Index of Interacting Fermions in One-Dimensional Superconductors
}

\author{
Dganit Meidan, ${ }^{1,2}$ Alessandro Romito, ${ }^{2}$ and Piet W. Brouwer ${ }^{2}$ \\ ${ }^{1}$ Department of Physics, Ben-Gurion University of the Negev, Beer-Sheva 84105, Israel \\ ${ }^{2}$ Dahlem Center for Complex Quantum Systems and Fachbereich Physik, Freie Universität Berlin, 14195 Berlin, Germany
}

(Received 22 December 2013; published 1 August 2014)

\begin{abstract}
We construct a scattering matrix formulation for the topological classification of one-dimensional superconductors with effective time-reversal symmetry in the presence of interactions. For an isolated system, Fidkowski and Kitaev have shown that such systems have a $\mathbb{Z}_{8}$ topological classification. We here show that these systems have a unitary scattering matrix at zero temperature when weakly coupled to a normal-metal lead, with a topological index given by the trace of the Andreev-reflection matrix, tr $r_{\text {he }}$. With interactions, $\operatorname{tr} r_{\text {he }}$ generically takes on the finite set of values $0, \pm 1, \pm 2, \pm 3$, and \pm 4 . We show that the two topologically equivalent phases with $\operatorname{tr} r_{\text {he }}= \pm 4$ support emergent many-body end states, which we identify to be a topologically protected Kondo-like resonance. The path in phase space that connects these equivalent phases crosses a non-Fermi-liquid fixed point where a multiple-channel Kondo effect develops. Our results connect the topological index to transport properties, thereby highlighting the experimental signatures of interacting topological phases in one dimension.
\end{abstract}

DOI: 10.1103/PhysRevLett.113.057003

Superconducting wires exist in two topologically distinct classes, which can be distinguished by the presence or absence of a Majorana bound state at the wire's ends [1-4]. Because of their non-Abelian exchange statistics [5-7], such Majorana states have been proposed as an element for a fault-tolerant quantum computational scheme [8-11]. The topological phases have been shown to be robust against the presence of (moderate) disorder [12,13] or interactions [14-17]. While only a few rather exotic systems are believed to realize the nontrivial phase $[5,18,19]$, such systems can in principle be engineered in solid state devices [20-22]. Recent experiments on a semiconductor wire proximity coupled to superconductors have been reported to show indications of the existence of Majorana bound states [23,24].

Whereas the topological classification of superconducting wires with or without Majorana states exclusively rests on the presence of particle-hole symmetry, in many of the proposals for the actual realization of topological superconducting wires an additional approximate effective timereversal symmetry appears [25]. For the $p+i p$ model of a spinless superconductor wire of width $L_{y}$ and Fermi velocity $v_{F}$, the effective time-reversal symmetry requires the superconducting gap to be much smaller than the transverse quantization energy $\hbar v_{F} / L_{y}$ [26], a condition that is easily met in view of the generic smallness of the gap in the proposals to engineer topological superconductors. With effective time-reversal symmetry, Majorana states carry a sign, such that Majorana states of the same sign coexist at the same end of the superconducting wire. As a result, the effective time-reversal symmetry changes the topological classification from $\mathbb{Z}_{2}$ to $\mathbb{Z}$, which counts the number of Majorana end states, with sign.
PACS numbers: 74.78.Na, 03.67.Lx, 73.63.Nm, 74.20.Rp

Unlike the $\mathbb{Z}_{2}$ classes, the $\mathbb{Z}$ effective time-reversal symmetry classification is not stable against the presence of interactions, even if the interactions preserve the symmetry [27]. In a seminal work, Fidkowski and Kitaev [28] showed that interactions break the free-fermion $\mathbb{Z}$ topological index down to $\mathbb{Z}_{8}$ (see also Refs. [29-32]). To understand this result, one notes that for the seven topological classes with $n=0, \pm 1, \pm 2$, and \pm 3 there exist realizations with fewer than two fermions at each wire's end (as two Majorana states combine into one fermion). Hence, no local interaction term is allowed for the low-energy sector, and the free-fermion classification remains valid. Fidkowski and Kitaev then showed that the two classes with $n= \pm 4$ can be adiabatically connected by a suitable interaction, so that eight inequivalent classes remain [33].

The experimental observation of Majorana states inevitably relies on the coupling of the topological superconductor to a normal-metal contact, the simplest detection scheme being through a measurement of the two-terminal Andreev conductance [23,24]. With effective time-reversal symmetry, but without interactions, the Andreev conductance $G$ at zero bias and zero temperature takes the quantized value $G=2|n| e^{2} / h$, if there are $n$ Majorana end states [34-36]. Distinguishing positive and negative $n$ requires a phasesensitive measurement of the Andreev-reflection matrix, using $\operatorname{tr} r_{\mathrm{he}}=n$ [37].

What is the Andreev-reflection signature of the $\mathbb{Z}_{8}$ topological classification for a superconducting wire in the effective time-reversal symmetry class, and what is the nature of the emergent end states if the wire is weakly coupled to a normal contact? In this Letter, we answer these questions, showing how the breakdown of the free-fermion 
$\mathbb{Z}$ classification to $\mathbb{Z}_{8}$ is reflected in the Andreev-reflection matrix $r_{\text {he }}$. Establishing the scattering properties of interacting topological phases highlights the experimental signatures of the emergent end states in conductance measurements or Josephson currents, thereby providing an important link between recent theoretical advances and future experimental work.

Our main results can be summarized as follows: For a normal lead weakly coupled to the superconducting wire, we find (i) that generically the scattering matrix is well defined and unitary at zero temperature, in spite of the presence of interactions in the superconducting wire, and (ii) that, in the presence of interactions, $\operatorname{tr} r_{\text {he }}$ is restricted to the values $0, \pm 1$, $\pm 2, \pm 3$, and \pm 4 . Exceptions to these rules do occur, but they form a set of measure zero and are unstable to perturbations. The topological phases with $-3 \leq \operatorname{tr} r_{\text {he }} \leq 3$ are topologically equivalent to their free-fermion analogues with $|n| \leq 3$ uncoupled Majorana end states. Conversely, we show that the phases with $\operatorname{tr} r_{\text {he }}= \pm 4$ are characterized by emergent manybody end states, which we identify as topologically protected Kondo-like resonances. These two configurations, which are both stable with respect to perturbations, are topologically equivalent in the presence of interactions. The path in phase space that connects these equivalent phases crosses a nonFermi-liquid fixed point associated with a multiple-channel Kondo problem, without closing the bulk excitation gap in the superconductor.

Model.-We derive these results in the framework of a multichannel Majorana chain [38] with an effective timereversal symmetry $\mathcal{T}^{2}=1$, corresponding to class BDI in the Cartan classification [39]. In the absence of interactions, the Majorana chain is described by the Hamiltonian

$$
\begin{aligned}
H_{S}= & \sum_{\alpha, j}\left(\Delta_{\alpha} d_{j+1, \alpha}^{\dagger} d_{j, \alpha}^{\dagger}-t d_{j+1, \alpha}^{\dagger} d_{j, \alpha}+\text { H.c. }\right) \\
& +\sum_{\alpha, j} \mu d_{j, \alpha}^{\dagger} d_{j, \alpha},
\end{aligned}
$$

where $t>0$ is the hopping parameter, $\mu$ is the chemical potential, and $d_{j, \alpha}$ is the annihilation operator for a fermion on site $j$ and channel $\alpha$. We consider $j=1,2, \ldots$, so that Eq. (1) describes a half-infinite wire beginning at $j=1$. We choose $\Delta_{\alpha} \neq 0$ to be real, so that $H_{S}$ is invariant under an effective antiunitary time-reversal symmetry operation $\mathcal{T}, \mathcal{T} d_{j, \alpha} \mathcal{T}^{-1}=d_{j, \alpha}$.

Equation (1) can be conveniently rewritten in terms of Majorana operators $\gamma_{j, \alpha}=d_{j, \alpha}+d_{j, \alpha}^{\dagger}$ and $\tilde{\gamma}_{j, \alpha}=$ $-i\left(d_{j, \alpha}-d_{j, \alpha}^{\dagger}\right)$. The model undergoes a topological phase transition at $|\mu|=2|t|$. The topological phase is characterized by two zero-energy Majorana end states for each channel, exponentially localized at opposite ends of the chain and separated by an energy gap from the bulk excitations. Since we consider a half-infinite chain, only the end state located near $j=1$ is of relevance for us. For positive $\Delta_{\alpha}$, the operator for this Majorana end state commutes with $\mathcal{T}$ and we denote it by $\gamma_{\alpha}$; for negative $\Delta_{\alpha}$, the end-state operator anticommutes with $\mathcal{T}$, and we write $\tilde{\gamma}_{\alpha}$. The effective time-reversal symmetry permits perturbations that couple Majorana end states of different type, $\mathcal{T} i \gamma_{\alpha} \tilde{\gamma}_{\beta} \mathcal{T}^{-1}=i \gamma_{\alpha} \tilde{\gamma}_{\beta}$, but forbids coupling between Majorana end modes of the same type. The system is therefore classified by a $\mathbb{Z}$ topological index, which counts the number $N$ of Majorana end states of " $\gamma$ " type minus the number $\tilde{N}$ of end states of " $\tilde{\gamma}$ " type. Without loss of generality, we take the channels $\alpha=1,2, \ldots, N$ to be of $\gamma$ type, whereas the remaining channels $\alpha=N+$ $1, \ldots, N+\tilde{N}$ are of $\tilde{\gamma}$ type.

Scattering matrix classification.-To study the scattering properties, we couple the semi-infinite wire to a halfinfinite normal lead. The Hamiltonian is given by

$$
H=H_{S}+H_{\text {lead }}+H_{T},
$$

where $H_{\text {lead }}$ is the Hamiltonian of the noninteracting ideal lead: $H_{\text {lead }}=\sum_{k, \alpha} \xi_{k} c_{k, \alpha}^{\dagger} c_{k, \alpha}$. Here $c_{k, \alpha}$ is the annihilation operator for an electron with momentum $k$ measured with respect to the Fermi point and channel index $\alpha$ and $\xi_{k}$ the corresponding kinetic energy. The term $H_{T}$ describes tunneling between the lead and the superconductor, $H_{T}=\sum_{k, \alpha} \tilde{t}_{T, \alpha} c_{k, \alpha}^{\dagger} d_{1, \alpha}+$ H.c., $\tilde{t}_{T, \alpha}$ being the tunneling matrix element of the chain $\alpha$. Considering that the superconducting wire is gapped in the bulk, we can project the tunneling Hamiltonian onto the low-energy sector consisting of the zero-energy Majorana end states:

$$
\begin{aligned}
\tilde{H}_{T}= & \frac{1}{2} \sum_{k}\left(\sum_{\alpha=1}^{N} t_{T, \alpha} c_{k, \alpha}^{\dagger} \gamma_{\alpha}+i t_{T, \alpha} \sum_{\alpha=N+1}^{N+\tilde{N}} c_{k, \tilde{\alpha}}^{\dagger} \tilde{\gamma}_{\tilde{\alpha}}\right) \\
& + \text { H.c., }
\end{aligned}
$$

where $t_{T, \alpha}$ are the effective tunneling couplings of the end states Majorana to the lead. As the system is gapped, no quasiparticle excitations are transmitted through the superconductor, and scattering processes are described by a unitary reflection matrix. In the BDI class, each Majorana end mode gives rise to perfect Andreev reflection, although the sign of the reflection amplitude is opposite for the two types of Majorana modes. The topological index is $n \equiv N-$ $N=\operatorname{tr} r_{\text {he }}[37,40]$, where $r_{\text {he }}$ is evaluated at zero energy.

Generalization to interacting systems.-We now generalize the above classification scheme to interacting systems. We consider weak short-range two-fermion interactions, so that we consider only low-energy states below the bulk superconducting gap. When restricted to the low-energy sector of the Majorana end states, the effective interaction Hamiltonian involves the end-state operators $\gamma_{\alpha}$ and $\tilde{\gamma}_{\alpha}$ only [41]. If all Majorana end states are of the $\gamma$ type, the most general interaction in the low-energy sector has the form

$$
H_{\text {int }}=\sum_{\alpha_{1}<\alpha_{2}<\alpha_{3}<\alpha_{4}} W_{\alpha_{1} \alpha_{2} \alpha_{3} \alpha_{4}} \gamma_{\alpha_{1}} \gamma_{\alpha_{2}} \gamma_{\alpha_{3}} \gamma_{\alpha_{4}},
$$

with $W_{\alpha_{1} \alpha_{2} \alpha_{3} \alpha_{4}}$ the strengths of the different interaction terms. This interaction Hamiltonian commutes with the effective time-reversal operator $\mathcal{T}$. If there are end states of 
both types, additional terms with four operators $\tilde{\gamma}$ or mixed terms with two operators of each type are also allowed by the effective time-reversal symmetry.

We now discuss how the inclusion of the interaction Hamiltonian affects a Majorana chain with free-fermion topological index $n$. We find that the cases $|n|<4,|n|=4$, and $|n|>4$ are qualitatively different and discuss these three cases separately.

The case $|n|<4$.- Since generic potential perturbations gap out pairs of Majorana states of opposite type, for weak interactions it is sufficient to limit our discussion to the "minimal" realizations of the topological phases, which have $\tilde{N}=0$, if the topological index $n=N$ is positive, and $N=0$, if $n=-\tilde{N}$ is negative. Since the low-energy interaction Hamiltonian $H_{\text {int }}=0$ if $N<4$ and $\tilde{N}=0$, or if $\tilde{N}<4$ and $N=0$ [see Eq. (4)], we immediately conclude that the Andreev-reflection signatures of these phases are unaffected by interactions, so that the reflection matrix is unitary and $\operatorname{tr} r_{\text {he }}=n$.

The case $|n|=4$. - The case $n= \pm 4$ is nontrivial, since already in its minimal realization it allows for a nontrivial interaction Hamiltonian $H_{\text {int }}=W \gamma_{1} \gamma_{2} \gamma_{3} \gamma_{4}$. By introducing the fermionic operators $f_{\uparrow}=(1 / 2)\left(\gamma_{1}+i \gamma_{2}\right), f_{\downarrow}=(1 / 2) \times$ $\left(\gamma_{3}+i \gamma_{4}\right)$, the low-energy interaction Hamiltonian for the case $n=4$ takes the form $H_{\text {int }}=-W\left(2 f_{\uparrow}^{\dagger} f_{\uparrow}-1\right) \times$ $\left(2 f_{\downarrow}^{\dagger} f_{\downarrow}-1\right)$. The interaction lifts the fourfold degeneracy of the zero-energy level of the free-fermion system and creates two doubly degenerate correlated states at energy $\pm W$. The situation with a degenerate level located at the end of the superconducting wire closely resembles a local impurity problem, and much insight can be gained by performing a mapping to the latter. For the sake of simplicity, we continue our analysis for the case of equal tunneling matrix elements $t_{T, \alpha}=t_{T}$ [42]. Hereto, the tunneling Hamiltonian is rewritten by using the operators $g_{L, \uparrow, k}=$ $2^{-1 / 2}\left(c_{k, 1}+i c_{k, 2}\right), g_{R, \uparrow,-k}=2^{-1 / 2}\left(-c_{k, 1}^{\dagger}-i c_{k, 2}^{\dagger}\right), g_{L, \downarrow, k}=$ $2^{-1 / 2}\left(c_{k, 3}+i c_{k, 4}\right)$, and $g_{R, \downarrow,-k}=2^{-1 / 2}\left(-c_{k, 3}^{\dagger}-i c_{k, 4}^{\dagger}\right)$, so that the resulting Hamiltonian takes the form of the symmetric Anderson model [43]

$$
\begin{aligned}
H= & \sum_{\beta=L, R} \sum_{k, \sigma=\uparrow, \downarrow}\left[\xi_{k} g_{\beta, \sigma, k}^{\dagger} g_{\beta, \sigma, k}+\frac{t_{T}}{2}\left(g_{\beta, \sigma, k}^{\dagger} f_{\sigma}+\text { H.c. }\right)\right] \\
& -W\left(2 f_{\uparrow}^{\dagger} f_{\uparrow}-1\right)\left(2 f_{\downarrow}^{\dagger} f_{\downarrow}-1\right) .
\end{aligned}
$$

In general, the scattering matrix of this Kondo-like problem is nonunitary due to inelastic spin flip processes. However, at zero temperature the system reaches a Fermi-liquid fixed point, unitarity is recovered due to the formation of a Kondo screening cloud, and the scattering matrix takes the simple form [44]

$$
S_{\sigma, \sigma^{\prime}}=\left.\delta_{\sigma, \sigma^{\prime}}\left(\begin{array}{ll}
r_{R R} & t_{R L} \\
t_{L R} & r_{L L}
\end{array}\right)\right|_{\sigma}=\left(\begin{array}{cc}
0 & -1 \\
-1 & 0
\end{array}\right) .
$$

Returning to the original basis with the lead operators $c_{k, \alpha}$, one finds that the normal reflection matrix $r_{\mathrm{ee}}=0$, whereas $r_{\text {he }}=\mathbb{1}_{4 \times 4}$. We conclude that at zero temperature the topological index follows the noninteracting formula $\operatorname{tr} r_{\text {eh }}=n=4$. As a physical consequence, a tunneling conductance experiment will show a zero-bias zerotemperature Andreev conductance $G=8 e^{2} / h$, which is the same conductance as without interactions. However, unlike in the noninteracting case, this quantized conductance peak is a consequence of a formation of a many-body Kondolike resonance at the end of the interacting superconductor, and it no longer signals the presence of four Majorana states. We can interpret this collective state, which is pinned to the Fermi energy, as the emergent edge state of the interacting superconductor.

The same analysis can be applied to the $n=-4$ case. It gives $r_{\mathrm{he}}=-\mathbb{1}_{4 \times 4}$ and $\operatorname{tr} r_{\mathrm{he}}=n=-4$. This implies that $\operatorname{tr} r_{\text {he }}$ takes different quantized values for $n=4$ and $n=-4$. How should this result be interpreted in light of the knowledge that, with interaction, classes with $n=4$ and with $n=-4$ are topologically equivalent [28]?

To find a scenario that resolves this paradox, we monitor the Andreev-reflection matrix along a path that connects the two classes $n=4$ and $n=-4$. The minimum channel number for a continuous interpolation between $n=4$ and $n=-4$ is 12 . For definiteness, we consider eight channels with positive $\Delta$, labeled $\alpha=1,2, \ldots, 8$, and four channels with negative $\Delta$, labeled $\alpha=9,10,11,12$, so that $N=8$ and $\tilde{N}=4$; see Fig. 1. For the interaction Hamiltonian we choose

$$
H_{\text {int }}(\theta)=W\left(H_{8} \sin \theta+H_{8}^{\prime} \cos \theta\right)
$$

where

$$
\begin{aligned}
H_{8}= & \gamma_{1} \gamma_{2} \gamma_{3} \gamma_{4}+\gamma_{5} \gamma_{6} \gamma_{7} \gamma_{8}+\gamma_{1} \gamma_{2} \gamma_{5} \gamma_{6}+\gamma_{3} \gamma_{4} \gamma_{7} \gamma_{8} \\
& -\gamma_{2} \gamma_{3} \gamma_{6} \gamma_{7}-\gamma_{1} \gamma_{4} \gamma_{5} \gamma_{8}+\gamma_{1} \gamma_{3} \gamma_{5} \gamma_{7}+\gamma_{3} \gamma_{4} \gamma_{5} \gamma_{6} \\
& +\gamma_{1} \gamma_{2} \gamma_{7} \gamma_{8}-\gamma_{2} \gamma_{3} \gamma_{5} \gamma_{8}-\gamma_{1} \gamma_{4} \gamma_{6} \gamma_{7}+\gamma_{2} \gamma_{4} \gamma_{6} \gamma_{8} \\
& -\gamma_{1} \gamma_{3} \gamma_{6} \gamma_{8}-\gamma_{2} \gamma_{4} \gamma_{5} \gamma_{7}
\end{aligned}
$$

and $H_{8}^{\prime}$ is obtained from $H_{8}$ by the substitution $\gamma_{\alpha} \rightarrow \gamma_{\alpha+4}$ for $\alpha=1,2,3,4$ and $\gamma_{\alpha} \rightarrow \tilde{\gamma}_{\alpha+4}$ for $\alpha=5,6,7,8$. As shown by Fidkowski and Kitaev, the Hamiltonian $H_{8}$ drives the eight Majorana end states in the first eight channels into a nondegenerate topologically trivial ground state [28]. Similarly, the Hamiltonian $H_{8}^{\prime}$ drives the eight Majorana states in the last eight channels into a nondegenerate trivial ground state. (Since the last eight channels have four Majorana states of each type, a potential term coupling the last eight channels would have the same effect.) For $\theta \rightarrow 0$ the system is effectively in the freefermion $n=4$ class analyzed previously, with low-energy interaction Hamiltonian $H_{\text {int }} \propto \gamma_{1} \gamma_{2} \gamma_{3} \gamma_{4}$, while for $\theta \rightarrow$ $\pi / 2$ it is in the opposite case $n=-4$, with low-energy interaction Hamiltonian $\propto \tilde{\gamma}_{9} \tilde{\gamma}_{10} \tilde{\gamma}_{11} \tilde{\gamma}_{12}$. Thus, the family of interaction Hamiltonians $H_{\text {int }}(\theta)$ with $0 \leq \theta \leq \pi / 2$ 
(a)

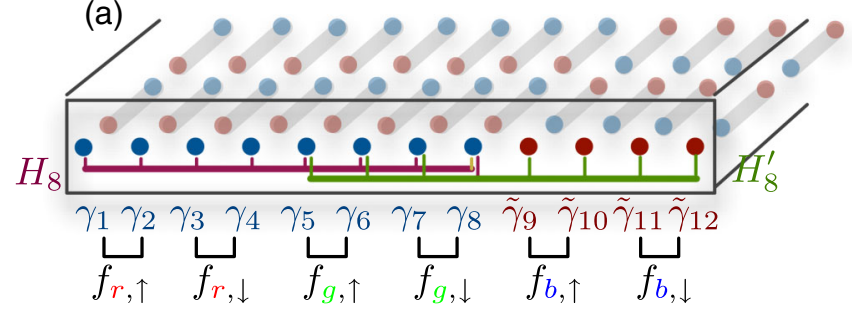

(b)
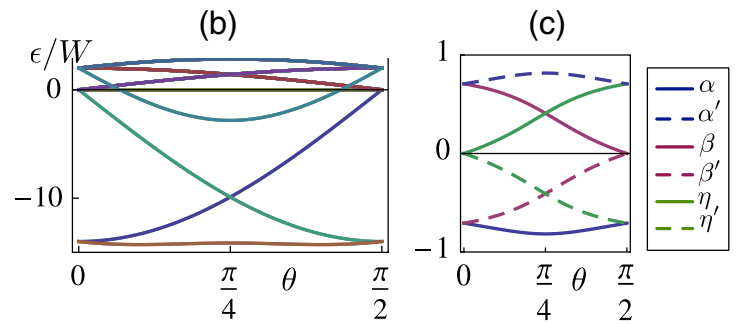

FIG. 1 (color online). (a) Schematic picture of 12 Majorana chains that interpolate between effective free-fermion classes $n=4$ and $n=-4$. The interactions terms coupling different Majorana end states are indicated by thick lines. The "colored" fermions constructed with Majorana fermions are indicated explicitly. (b) Energy spectrum as a function of the interpolation parameter $\theta$, showing that the spectrum remains gapped through the interpolation. The ground state is twofold degenerate. (c) Amplitudes $\alpha, \alpha^{\prime}, \beta, \beta^{\prime}, \eta$, and $\eta^{\prime}$ for the ground state wave functions, as a function of the interpolation parameter $\theta$; see Eq. (9).

smoothly interpolates between the (free-fermion) classes $n=4$ and $n=-4$.

To continue our analysis, we construct six fermionic operators out of the 12 Majorana operators, which we group into three "colors" $(r, g, b): f_{r, \uparrow}=(1 / 2)\left(\gamma_{1}+i \gamma_{2}\right)$, $f_{r, \downarrow}=(1 / 2)\left(\gamma_{3}+i \gamma_{4}\right), \quad f_{g, \uparrow}=(1 / 2)\left(\gamma_{5}+i \gamma_{6}\right), \quad f_{g, \downarrow}=$ $(1 / 2)\left(\gamma_{7}+i \gamma_{8}\right), \quad f_{b, \uparrow}=(1 / 2)\left(\tilde{\gamma}_{9}+i \tilde{\gamma}_{10}\right), \quad$ and $f_{b, \downarrow}=$ $(1 / 2)\left(\tilde{\gamma}_{11}+i \tilde{\gamma}_{12}\right)$; see Fig. 1. Diagonalization of the interaction Hamiltonian (7) reveals that the many-particle ground state is twofold degenerate for all $0<\theta<\pi / 2$ and confirms that the excitation spectrum remains gapped otherwise; see Fig. 1. The twofold degenerate ground state is spanned by the two states of the form

$$
\begin{gathered}
|\psi\rangle=\alpha|111111\rangle+\beta|110000\rangle+\eta|000011\rangle, \\
\left|\psi^{\prime}\right\rangle=\alpha^{\prime}|000000\rangle+\beta^{\prime}|001111\rangle+\eta^{\prime}|111100\rangle,
\end{gathered}
$$

where we use the basis of the occupations numbers $\left|n_{r \uparrow} n_{r \downarrow} n_{g \uparrow} n_{g \downarrow} n_{b \uparrow} n_{b \downarrow}\right\rangle$. The excitation gap and the real coefficients $\alpha, \alpha^{\prime}, \beta, \beta^{\prime}, \eta$, and $\eta^{\prime}$ as a function of $\theta$ are shown in Fig. 1. For $\theta \rightarrow 0^{+}$, we have $\eta=\eta^{\prime}=0$, and a transition between the two ground states is possible only by the exchange of two "red" fermions with two lead fermions in the first four channels $\alpha=1,2,3,4$. In this case, the system can be mapped to the symmetric Anderson model, as discussed above. Hence, at zero temperature, the reflection matrix is unitary and satisfies $\operatorname{tr} r_{\mathrm{eh}}=4$. Conversely, for $\theta \rightarrow \pi / 2^{-}$, we find $\beta=\beta^{\prime}=0$, and a transition between the two ground states is possible only by the exchange of two "blue" fermions with lead channels in the last set of four channels, $\alpha=9,10,11,12$. In this case, the system is again mapped to a symmetric Anderson model, but with $\operatorname{tr} r_{\mathrm{eh}}=-4$ at zero temperature. For $0<\theta<\pi / 2$, generically all six amplitudes in Eq. (9) are nonzero. In this case, transitions between the two ground states can take place by the exchange of two fermions of arbitrary but equal colors. Since there are three colors in total, the model is mapped to a three-channel Kondo problem. Such a multichannel Kondo problem is, however, unstable, and the system flows to a single-channel Kondo fixed point determined by the strongest coupling constant and correspondingly to one of the two extreme limits discussed above. Upon increasing $\theta$ from 0 to $\pi / 2$, invariably there must be a point at which the coupling to the "blue" channels and the "red" or "green" channels is equal. This point is associated with a sharp phase boundary that exhibits a non-Fermi-liquid behavior, due to the formation of a multiple- (generically, two-) channel Kondo state at the wire's end. It follows that the scattering matrix goes through a nonunitary point along the path connecting these two phases. It is at this point that the transition between the quantized values $\operatorname{tr} r_{\text {he }}= \pm 4$ can take place. We note that this observation is consistent with recent studies that show that the crossover between topologically distinct noninteracting classes that become equivalent when interactions are present is associated with zeros of the Green's function indicating the formation of a non-Fermi-liquid state [32].

The case $|n|>4$.-Upon including interactions, the free-fermion cases with $|n|=5,6,7$, or 8 can be continuously connected to free-fermion classes with $|n|=3,2$, 1 , and 0 , respectively. An explicit example of a generic low-energy Hamiltonian that interpolates between the freefermion phases of $n=7$ and $n=-1$ is presented in Supplemental Material [45]. An analysis of the transitions between the two degenerate ground states reveals that the free-fermion $n=7$ configuration is unstable to interactions and that in the weak-coupling limit $\left|t_{T}\right| \ll|t|$ the interacting system flows to an effectively noninteracting configuration with $\operatorname{tr} r_{\text {he }}=-1$. A similar analysis can be applied to $|n|=5,|n|=6$, and $|n|=8$.

Conclusions.-We have constructed a scattering matrix formulation for the topological index of interacting fermions in one dimension with an effective time-reversal symmetry. The scattering matrix of the interacting system is unitary at zero temperature and zero energy, and the topological index can be calculated from the trace tr $r_{\text {he }}$. With interactions, the topological index is restricted to nine possible values: $\operatorname{tr} r_{\mathrm{he}}=0, \pm 1, \pm 2, \pm 3$, and \pm 4 . Whereas the phases with $\left|\operatorname{tr} r_{\text {he }}\right|<4$ are effectively single-particle phases, we have shown that the two topologically equivalent phases with $\operatorname{tr} r_{\mathrm{he}}= \pm 4$ are characterized by emergent many-body end states, which we identify to be a topologically protected Kondo-like resonance. The path in phase space that connects these equivalent phases crosses a nonFermi-liquid fixed point where a multiple-channel Kondo effect develops. 
Although the main motivation for our work is fundamental, a theory of implications of the topological classification for scattering properties is essential for a theoretical description of experimental geometries, where topological superconductors necessarily need to be connected to normal-metal probes. The effective time-reversal symmetry that is behind the $\mathbb{Z}_{8}$ topological classification is relevant for some of the recent proposals to realize topological superconductors in one dimension. Whereas interactions are believed to be of minor importance if the topological superconductivity derives from the proximity of a bulk superconductor, experimental efforts to minimizing the electric screening of nearby superconductors in order to allow for local gating of the device inevitably lead to a larger role of interactions in the topological superconductor.

We gratefully acknowledge discussions with Yigal Meir and Michele Filippone. This work is supported by the Alexander von Humboldt Foundation.

[1] M. Z. Hasan and C. L. Kane, Rev. Mod. Phys. 82, 3045 (2010).

[2] X.-L. Qi and S.-C. Zhang, Rev. Mod. Phys. 83, 1057 (2011).

[3] J. Alicea, Rep. Prog. Phys. 75, 076501 (2012).

[4] T. D. Stanescu and S. Tewari, J. Phys. Condens. Matter 25, 233201 (2013).

[5] G. Moore and N. Read, Nucl. Phys. B360, 362 (1991).

[6] N. Read and D. Green, Phys. Rev. B 61, 10267 (2000).

[7] D. A. Ivanov, Phys. Rev. Lett. 86, 268 (2001).

[8] A. Kitaev, Ann. Phys. (Amsterdam) 303, 2 (2003).

[9] M. H. Freedman, Proc. Natl. Acad. Sci. U.S.A. 95, 98 (1998).

[10] A. Kitaev, Ann. Phys. (Amsterdam) 321, 2 (2006).

[11] C. Nayak, S. H. Simon, A. Stern, M. Freedman, and S. Das Sarma, Rev. Mod. Phys. 80, 1083 (2008).

[12] O. Motrunich, K. Damle, and D. A. Huse, Phys. Rev. B 63, 224204 (2001).

[13] P. W. Brouwer, M. Duckheim, A. Romito, and F. von Oppen, Phys. Rev. Lett. 107, 196804 (2011).

[14] S. Gangadharaiah, B. Braunecker, P. Simon, and D. Loss, Phys. Rev. Lett. 107, 036801 (2011).

[15] L. Fidkowski, R. M. Lutchyn, C. Nayak, and M. P. A. Fisher, Phys. Rev. B 84, 195436 (2011).

[16] E. M. Stoudenmire, J. Alicea, O. A. Starykh, and M. P. A. Fisher, Phys. Rev. B 84, 014503 (2011).

[17] E. Sela, A. Altland, and A. Rosch, Phys. Rev. B 84, 085114 (2011).

[18] G. E. Volovik, The Universe in a Helium Droplet (Oxford University, New York, 2003).

[19] S. Das Sarma, C. Nayak, and S. Tewari, Phys. Rev. B 73, 220502 (2006).

[20] L. Fu and C. L. Kane, Phys. Rev. Lett. 100, 096407 (2008).

[21] R. M. Lutchyn, J. D. Sau, and S. Das Sarma, Phys. Rev. Lett. 105, 077001 (2010).

[22] Y. Oreg, G. Refael, and F. von Oppen, Phys. Rev. Lett. 105, 177002 (2010).

[23] V. Mourik, K. Zuo, S. M. Frolov, S. R. Plissard, E. P. A. M. Bakkers, and L.P. Kouwenhoven, Science 336, 1003 (2012).
[24] A. Das, Y. Ronen, Y. Most, Y. Oreg, M. Heiblum, and H. Shtrikman, Nat. Phys. 8, 887 (2012).

[25] S. Tewari and J. D. Sau, Phys. Rev. Lett. 109, 150408 (2012).

[26] G. Kells, D. Meidan, and P. W. Brouwer, Phys. Rev. B 85, 060507 (2012).

[27] See Supplemental Material at http://link.aps.org/ supplemental/10.1103/PhysRevLett.113.057003, residual Coulomb interactions are sufficient to induce interactions between Majorana end states, despite the fact that the Majorana end states are neutral.

[28] L. Fidkowski and A. Kitaev, Phys. Rev. B 81, 134509 (2010).

[29] A. M. Turner, F. Pollmann, and E. Berg, Phys. Rev. B 83, 075102 (2011).

[30] L. Fidkowski and A. Kitaev, Phys. Rev. B 83, 075103 (2011).

[31] V. Gurarie, Phys. Rev. B 83, 085426 (2011).

[32] S. R. Manmana, A. M. Essin, R. M. Noack, and V. Gurarie, Phys. Rev. B 86, 205119 (2012).

[33] To be precise, Ref. [28] proves the equivalent statement that the classes with $n=0$ and $n=8$ are adiabatically connected. An example of a continuous connection between $n=4$ and $n=-4$ is given in this article.

[34] K. T. Law, P. A. Lee, and T. K. Ng, Phys. Rev. Lett. 103, 237001 (2009).

[35] K. Flensberg, Phys. Rev. B 82, 180516(R) (2010).

[36] Higher values of the Andreev conductance are possible in principle, but these are unstable to perturbations that preserve the effective time-reversal symmetry.

[37] I. C. Fulga, F. Hassler, A. R. Akhmerov, and C. W. J. Beenakker, Phys. Rev. B 83, 155429 (2011).

[38] A. Y. Kitaev, Phys. Usp. 44, 131 (2001).

[39] A. Altland and M. R. Zirnbauer, Phys. Rev. B 55, 1142 (1997).

[40] M.-T. Rieder, P. W. Brouwer, and I. Adagideli, Phys. Rev. B 88, 060509 (2013).

[41] A different setup, in which nonlocal charging effects couple end states at opposite ends of a Majorana chain, gives rise to a "topological Kondo effect"; see B. Béri and N. R. Cooper, Phys. Rev. Lett. 109, 156803 (2012); A. Altland and R. Egger, Phys. Rev. Lett. 110, 196401 (2013); B. Béri, Phys. Rev. Lett. 110, 216803 (2013).

[42] See Supplemental Material at http://link.aps.org/ supplemental/10.1103/PhysRevLett.113.057003 for the analysis of the case of generic tunneling matrix elements.

[43] Note that it is the effective time-reversal symmetry in the original formulation of the problem that protects this symmetric point and that no fine-tuning is needed to arrive at the Hamiltonian (5).

[44] T. K. Ng and P. A. Lee, Phys. Rev. Lett. 61, 1768 (1988).

[45] See Supplemental Material at http://link.aps.org/ supplemental/10.1103/PhysRevLett.113.057003, which includes Refs. [46-49].

[46] A. C. Hewson, The Kondo Problem to Heavy Fermions (Cambridge University Press, Cambridge, England, 1997).

[47] P. Coleman and A. J. Schofield, Phys. Rev. Lett. 75, 2184 (1995).

[48] P. Coleman, L. B. Ioffe, and A. M. Tsvelik, Phys. Rev. B 52, 6611 (1995).

[49] M. Fabrizio, A. O. Gogolin, and P. Nozières, J. Supercond. 9, 425 (1996). 